\title{
OPEN Analysis of serious adverse events in a pediatric community-acquired pneumonia randomized clinical trial in Malawi
}

\author{
Amy Sarah Ginsburg ${ }^{\bowtie}$ \& Susanne May
}

Amoxicillin is recommended as first-line antibiotic treatment for community-acquired pneumonia, the leading infectious cause of mortality in children aged less than 5 years. We conducted a double-blind, randomized controlled non-inferiority trial comparing 3- to 5-day amoxicillin treatment for non-severe chest-indrawing pneumonia in HIV-negative children aged 2 to 59 months in Malawi. In a secondary analysis, we assessed the frequency of serious adverse events (SAEs) during the trial to evaluate the safety of treatment with amoxicillin. Enrolled children with non-severe chest-indrawing pneumonia were randomized to either 3- or 5-day amoxicillin and followed for 14 days to track clinical outcomes. In addition to evaluation for treatment failure (primary endpoint, day 6), relapse, and study drug adherence, children were assessed for adverse events, including SAEs, which were managed per local standard clinical practice until resolution or stabilization. Between March 2016 and April 2019, 3000 children were enrolled, with male and younger children (aged less than 24 months) demonstrating more SAEs $(10.3 \%$ for males vs $8.1 \%$ for females, $p=0.04 ; 10.0 \%$ for $2-6$ months, $10.8 \%$ for 7-11 months, $9.7 \%$ for $12-23$ months and 5.6\% for $24-59$ months, $p=0.01$ ). The most common SAEs were progression of or recurrent pneumonia (220 SAEs in 217 children), acute gastroenteritis (14 SAEs in 14 children), and fever (8 SAEs in 8 children); however, there were no significant or substantive differences in the percentage of children with pneumonia-related, acute gastroenteritis, or fever SAEs noted between the 3-versus 5-day amoxicillin treatment groups. In our pediatric community-acquired pneumonia trial evaluating amoxicillin treatment, there were relatively few SAEs overall and very few attributed to amoxicillin. Duration of amoxicillin treatment did not impact the frequency of SAEs. We found male and younger children appear to be more vulnerable to SAEs in our trial; however, our data support previous data demonstrating the safety of amoxicillin use in children with pneumonia.

Clinical trial registration: ClinicalTrials.gov (NCT02678195).

Acute respiratory tract infections are the most common reason for patient encounters in pediatric care and for antibiotic prescriptions ${ }^{1,2}$. Of all medication classes prescribed to children in both hospital and community settings, antibiotics are responsible for the majority of adverse drug events ${ }^{3}$. In addition to being one of the most commonly prescribed antibiotics globally, amoxicillin accounts for among the most frequent adverse drug events seen in emergency departments ${ }^{1,2,4}$. Amoxicillin is recommended as first-line antibiotic treatment of communityacquired pneumonia, the leading infectious cause of mortality in children less than five years of age $\mathrm{s}^{5}$ Generally well-tolerated and safe, amoxicillin has important side effects which include diarrhea, nausea, vomiting, fever, and rash as well as rarer but more serious side effects such as abnormal liver function tests, interstitial nephritis, seizures, and Stevens-Johnson syndrome, all of which could lead to serious adverse events (SAEs) ${ }^{6-9}$. To evaluate the safety of treatment with amoxicillin in children enrolled in a pediatric community-acquired pneumonia treatment trial, we conducted a secondary analysis and assessed the frequency of SAEs. 


\section{Methods}

In a prospective double-blind randomized controlled non-inferiority clinical trial carried out in a malariaendemic region of Malawi, we enrolled HIV-uninfected children aged 2 to 59 months with non-severe chestindrawing pneumonia and compared treatment with 5-day versus 3-day oral amoxicillin dispersible tablets (Appendix 1. Study protocol) $^{10}$. Following initial study drug administration, children were typically observed in-hospital for 2 days, discharged on day 3 if no treatment failure criteria (World Health Organization general danger sign (lethargy or unconsciousness, convulsions, vomiting everything, inability to drink or breastfeed), sign of severe respiratory distress (grunting, nasal flaring, head nodding, or chest indrawing), or hypoxemia) were present, and followed for 14 days to track clinical outcomes. At all scheduled (days 2, 4, 6, and 14), and unscheduled follow-up visits, children were assessed for treatment failure (primary endpoint, day 6 with a relative non-inferiority margin of 1.5 times the treatment failure rate in the 5-day amoxicillin group) or relapse, and study drug adherence ${ }^{11}$. All adverse events were assessed and managed per local standard clinical practice, documented, and followed and treated until resolution or stabilization. All SAEs were reported to the study safety team for review within $24 \mathrm{~h}$ and a detailed SAEs report was completed. The United States National Institutes of Health Division of AIDS adverse event grading system was used to assess severity of the event ${ }^{12}$. For continuous baseline characteristics, averages between children reporting any SAEs versus children reporting no SAEs were compared using t-tests. Differences in the number of children who reported SAEs for different baseline characteristics as well as safety outcomes were assessed using chi-square statistics or, if the expected cell count was less than five, Fisher's exact tests. No adjustments were made for multiple comparisons and complete case analyses were used. A data safety monitoring board was established to routinely and independently assess child safety throughout the trial. The trial was approved by Western Institutional Review Board; College of Medicine Research and Ethics Committee; and Malawi Pharmacy, Medicines and Poisons Board; and was registered with ClinicalTrials.gov (NCT02678195; 09/02/2016).

Ethical approval and consent to participate. The study was conducted in accordance with the International Conference on Harmonisation, Good Clinical Practice and the Declaration of Helsinki 2008, and was approved by the Western Institutional Review Board in the state of Washington, USA; the College of Medicine Research and Ethics Committee, Blantyre, Malawi; and the Malawi Pharmacy, Medicines and Poisons Board. Written informed consent for study participation was obtained from the caregiver or legal guardian of each study participant.

\section{Results}

Conducted between March 2016 and April 2019 at Kamuzu Central Hospital and Bwaila District Hospital in Lilongwe, Malawi, 3000 children were enrolled and included in this analysis. As reported previously, children receiving 3 days of amoxicillin had a 5.9\% (85/1442 with outcome data) treatment failure rate by Day 6, within the non-inferiority margin of those receiving 5 days of amoxicillin $(5.2 \%(75 / 1456)$ treatment failure rate), with an adjusted absolute difference of $0.75 \%$ and $95 \%$ confidence interval (CI) $-0.92 \%, 2.41 \%{ }^{10}$. In addition to presenting with more severe pneumonia, male and younger children (aged less than 24 months) demonstrated more SAEs ( $10.3 \%$ for males vs $8.1 \%$ for females, $p=0.04 ; 10.0 \%$ for $2-6$ months, $10.8 \%$ for $7-11$ months, $9.7 \%$ for $12-23$ months and $5.6 \%$ for $24-59$ months, $p=0.01$ ) (Table 1). The most common SAEs were progression or recurrent pneumonia (220 SAEs in 217 children), acute gastroenteritis (14 SAEs in 14 children), and fever (8 SAEs in 8 children) (Table 2); however, there were no significant or substantive differences in the percentage of children with pneumonia-related, acute gastroenteritis, or fever SAEs noted between the 5- versus 3-day amoxicillin treatment groups. Furthermore, when comparing the grade of SAE severity between the amoxicillin treatment groups, there were no significant or substantive differences noted (Table 3). Six children had life-threatening SAEs (all with danger sign pneumonia on days 1, 2 or 3 ) and three children died (one with pneumonia on day 5 , one with danger sign pneumonia on day 5 , and one with gastroenteritis on day 11). Nine children had two SAEs and no child had more than two SAEs. Only two SAEs were determined to be possibly (one with danger sign pneumonia) or probably (one with fever) related to amoxicillin.

\section{Discussion}

In our pediatric community-acquired pneumonia trial evaluating amoxicillin treatment, there were relatively few SAEs overall and very few attributed to amoxicillin. In addition to finding 3 days of amoxicillin treatment for chest-indrawing pneumonia non-inferior to 5 days among HIV-uninfected Malawian children, the duration of amoxicillin treatment did not appear to impact the frequency of SAEs. The low rate of SAEs may be partly a consequence of the high level of supportive care and monitoring the enrolled children received and/or of the study eligibility criteria that excluded HIV-seropositive children and those with signs of severe disease or acute malnutrition. However, since common harms from antibiotics are poorly quantified and frequently not reported, reviewing SAEs frequency is important to assess potential serious adverse impacts of amoxicillin used for treatment of non-severe chest-indrawing pediatric pneumonia.

In our trial, we found male and younger children appear to be more vulnerable to SAEs. Male sex and younger age have been shown to be associated with more severe disease presentations and increased mortality ${ }^{13,14}$. Furthermore, amoxicillin adverse events have shown to be higher for younger children ${ }^{4}$. Contributing factors may 


\begin{tabular}{|c|c|c|c|}
\hline \multirow[b]{2}{*}{ Overall } & \multicolumn{2}{|c|}{$\begin{array}{l}\text { Number with SAEs } \\
\text { or average by SAE } \\
\text { status }\end{array}$} & \multirow[t]{2}{*}{$p$ value } \\
\hline & $279 / 3000$ & $9.3 \%$ & \\
\hline \multicolumn{4}{|l|}{ Sex } \\
\hline Males & $170 / 1653$ & $10.3 \%$ & 0.04 \\
\hline Females & $109 / 1347$ & $8.1 \%$ & \\
\hline \multicolumn{3}{|l|}{ Age (years) } & 0.01 \\
\hline $2-6$ & $116 / 1155$ & $10.0 \%$ & \\
\hline $7-11$ & $63 / 581$ & $10.8 \%$ & \\
\hline $12-23$ & $69 / 713$ & $9.7 \%$ & \\
\hline $24-59$ & $31 / 551$ & $5.6 \%$ & \\
\hline \multicolumn{3}{|c|}{ Respiratory rate (breaths/min) } & 0.37 \\
\hline$<40$ & $28 / 406$ & $6.9 \%$ & \\
\hline $40-49$ & $100 / 1034$ & $9.7 \%$ & \\
\hline $50-59$ & $100 / 1090$ & $9.2 \%$ & \\
\hline $60-69$ & $45 / 416$ & $10.8 \%$ & \\
\hline$\geq 70$ & $6 / 54$ & $11.1 \%$ & \\
\hline \multicolumn{3}{|l|}{ Oxygen saturation (\%) } & 0.38 \\
\hline$<90$ & 0 & 0 & \\
\hline $90-92$ & $2 / 10$ & $16.7 \%$ & \\
\hline$\geq 93$ & $277 / 2988$ & $9.3 \%$ & \\
\hline \multicolumn{3}{|c|}{ Axillary temperature $\left({ }^{\circ} \mathrm{C}\right)$} & 0.49 \\
\hline$<38$ & $198 / 2074$ & $9.6 \%$ & \\
\hline$\geq 38$ & $81 / 926$ & $8.8 \%$ & \\
\hline Heart rate (beats/min) & Mean (SD) & & 0.31 \\
\hline Without SAEs (2721) & $150.2(16.3)$ & & \\
\hline With SAEs (279) & $151.3(14.9)$ & & \\
\hline \multicolumn{3}{|c|}{ Weight-for-height z-score } & 0.95 \\
\hline Without SAEs (2721) & $0.83(1.3)$ & & \\
\hline With SAEs (279) & $0.82(1.3)$ & & \\
\hline \multicolumn{3}{|c|}{ Mid-upper arm circumference $(\mathrm{cm})$} & 0.11 \\
\hline$\leq 12.5$ & $18 / 136$ & $13.2 \%$ & \\
\hline$>12.5$ & $261 / 2864$ & $9.1 \%$ & \\
\hline \multicolumn{3}{|c|}{ Malaria (rapid diagnostic test) } & 0.38 \\
\hline Yes & $21 / 269$ & $7.8 \%$ & \\
\hline No & $258 / 2731$ & $9.5 \%$ & \\
\hline \multicolumn{3}{|l|}{ Diarrhea } & 0.77 \\
\hline Yes & $40 / 413$ & $9.7 \%$ & \\
\hline No & $239 / 2587$ & $9.2 \%$ & \\
\hline
\end{tabular}

Table 1. Number of children for whom serious adverse events (SAEs) were reported by baseline characteristics.

include increased susceptibility to antibiotic allergy among younger children and caregivers having a lower threshold for seeking care for young children they perceive to be more vulnerable ${ }^{15}$.

Limitations to our study included the small sample size of children with SAEs and the limited duration of follow-up ( 2 weeks). Potential bias may have been introduced with inaccurate self-reporting of adherence which, if present, could have resulted in making the groups look more similar for primary, secondary, and safety outcomes. In addition, the study did not obtain laboratory or imaging confirmation regarding the diagnosis of chestindrawing pneumonia. As a result, we may have observed fewer SAEs than would have been the case otherwise.

While our clinical trial data further support previous data demonstrating the safety of amoxicillin use in children with pneumonia ${ }^{16-18}$, we believe it is important to ensure adequate and complete safety monitoring and to report and disseminate the findings, particularly from studies involving children. The dissemination of these data as well as active and passive surveillance data that help quantify and qualify the benefits versus risks of antibiotic treatment in young children is critical to better understanding the optimal use cases in young children. 


\begin{tabular}{|c|c|c|c|}
\hline & 3 days amoxicillin & 5 days amoxicillin & $p$ value \\
\hline \multicolumn{3}{|c|}{ Fast-breathing pneumonia } & 0.58 \\
\hline No & 1480 & 1489 & \\
\hline Yes & 17 & 14 & \\
\hline \multicolumn{3}{|c|}{ Chest-indrawing pneumonia } & 0.27 \\
\hline No & 1437 & 1454 & \\
\hline Yes & 60 & 49 & \\
\hline \multicolumn{3}{|c|}{ Danger sign pneumonia } & 0.86 \\
\hline No & 1448 & 1452 & \\
\hline Yes & 49 & 51 & \\
\hline \multicolumn{3}{|c|}{ Chest $x$-ray confirmed pneumonia } & 0.20 \\
\hline No & 1490 & 1500 & \\
\hline Yes & 7 & 3 & \\
\hline \multicolumn{3}{|c|}{ Acute gastroenteritis } & 0.59 \\
\hline No & 1489 & 1497 & \\
\hline Yes & 8 & 6 & \\
\hline \multicolumn{3}{|c|}{ Fever } & $0.73^{*}$ \\
\hline No & 1494 & 1498 & \\
\hline Yes & 3 & 5 & \\
\hline
\end{tabular}

Table 2. Number of children with serious adverse events (SAEs) by treatment group. ${ }^{\star}$ Based on Fisher's exact test.

\begin{tabular}{|c|c|c|c|}
\hline & 3 days amoxicillin & 5 days amoxicillin & Total \\
\hline Highest grade (per child) $(p=0.20)^{*}$ & 147 & 132 & 279 \\
\hline Mild-1 & 0 & 3 & 3 \\
\hline Moderate-2 & 20 & 17 & 37 \\
\hline Severe-3 & 121 & 108 & 229 \\
\hline Life-threatening -4 & 5 & 1 & 6 \\
\hline Death-5 & 1 & 2 & 3 \\
\hline Missing & 0 & 1 & 1 \\
\hline Relatedness of SAE $E^{* *}$ & 3 days amoxicillin & 5 days amoxicillin & \\
\hline Not related & 146 & 129 & \\
\hline Probably not related & 1 & 0 & \\
\hline Possibly related & 0 & 1 & \\
\hline Probably related & 0 & 1 & \\
\hline Missing & 0 & 1 & \\
\hline
\end{tabular}

Table 3. Characteristics of serious adverse events (SAEs) by treatment group. ${ }^{\star}$ Based on Fisher's exact test. ${ }^{*}$ No formal testing done because of small numbers.

Received: 9 July 2021; Accepted: 21 February 2022

Published online: 03 March 2022

\section{References}

1. Rogawski, E. T. et al. Use of antibiotics in children younger than two years in eight countries: A prospective cohort study. Bull: World Health Organ. 95(1), 49-61 (2017).

2. Klein, E. Y. et al. Global increase and geographic convergence in antibiotic consumption between 2000 and 2015 . Proc. Natl. Acad. Sci. U. S. A. 115(15), E3463-E3470 (2018).

3. Smyth, R. M. et al. Adverse drug reactions in children-A systematic review. PLoS ONE 7(3), e24061 (2012).

4. Lovegrove, M. C. et al. US emergency department visits for adverse drug events from antibiotics in children, 2011-2015. J. Pediatr. Infect. Dis. Soc. 8(5), 384-391 (2019).

5. World Health Organization (WHO). Revised WHO classification and treatment of pneumonia in children at health facilities: evidence summaries. (World Health Organization, 2014).

6. FDA. Amoxil. Available: https://www.accessdata.fda.gov/drugsatfda_docs/label/2008/050760s11,050761s11,050754s12,050542s251 bl.pdf

7. LiverTox: Clinical and Research Information on Drug-Induced Liver Injury (2012).

8. Gillies, M. et al. Common harms from amoxicillin: A systematic review and meta-analysis of randomized placebo-controlled trials for any indication. CMAJ 187(1), E21-E31 (2015). 
9. Nkwopara, E. et al. Analysis of serious adverse events in a paediatric fast breathing pneumonia clinical trial in Malawi. BMJ Open Respir. Res. 6(1), e000415 (2019).

10. Ginsburg, A. S. et al. Amoxicillin for 3 or 5 days for chest-indrawing pneumonia in Malawian children. N. Engl. J. Med. 383(1), 13-23 (2020).

11. Ginsburg, A. S. \& May, S. Adherence to oral amoxicillin dispersible tablets in children with community-acquired pneumonia enrolled in clinical trials in Malawi. Pneumonia (Nathan) 13(1), 10 (2021).

12. U.S. Department of Health and Human Services, National Institutes of Health, National Institute of Allergy and Infectious Diseases, Division of AIDS. Division of AIDS (DAIDS) Table for Grading the Severity of Adult and Pediatric Adverse Events, Corrected Version 2.1. [July 2017]. Available from: https://rsc.niaid.nih.gov/sites/default/files/daidsgradingcorrectedv21.pdf

13. Ginsburg, A. S. \& May, S. Fast-breathing vs chest-indrawing childhood pneumonia: Baseline characteristics. Int. J. Infect. Dis. 104, 361-362 (2021).

14. le Roux, D. M. et al. Lower respiratory tract infections in children in a well-vaccinated South African birth cohort: Spectrum of disease and risk factors. Clin Infect Dis. 69(9), 1588-1596 (2019).

15. Marrs, T., Fox, A. T., Lack, G. \& du Toit, G. The diagnosis and management of antibiotic allergy in children: Systematic review to inform a contemporary approach. Arch. Dis. Child. 100(6), 583-588 (2015).

16. Addo-Yobo, E. et al. Outpatient treatment of children with severe pneumonia with oral amoxicillin in four countries: The MASS study. Trop. Med. Int. Health 16(8), 995-1006 (2011).

17. Sadruddin, S. et al. Comparison of 3 days amoxicillin versus 5 days co-trimoxazole for treatment of fast-breathing pneumonia by community health workers in children aged 2-59 months in Pakistan: A cluster-randomized trial. Clin. Infect. Dis. 69(3), 397-404 (2019).

18. Shahrin, L. et al. Intravenous amoxicillin plus intravenous gentamicin for children with severe pneumonia in Bangladesh: An open-label, randomized, non-inferiority controlled trial. Life (Basel) 11(12), 1299 (2021).

\section{Author contributions}

A.S.G. conceptualized the study, obtained research funding, designed the study and data collection instruments, coordinated and supervised data collection from the site, interpreted the data, and drafted the manuscript. S.M. designed the study, performed the statistical analyses and interpreted the data, and drafted sections of the manuscript. Both authors worked collaboratively to review and revise the manuscript and agree to be accountable for the work.

\section{Funding}

This work was funded by a grant from the Bill and Melinda Gates Foundation (OPP1105080).

\section{Competing interests}

The authors declare no competing interests.

\section{Additional information}

Correspondence and requests for materials should be addressed to A.S.G.

Reprints and permissions information is available at www.nature.com/reprints.

Publisher's note Springer Nature remains neutral with regard to jurisdictional claims in published maps and institutional affiliations.

Open Access This article is licensed under a Creative Commons Attribution 4.0 International License, which permits use, sharing, adaptation, distribution and reproduction in any medium or format, as long as you give appropriate credit to the original author(s) and the source, provide a link to the Creative Commons licence, and indicate if changes were made. The images or other third party material in this article are included in the article's Creative Commons licence, unless indicated otherwise in a credit line to the material. If material is not included in the article's Creative Commons licence and your intended use is not permitted by statutory regulation or exceeds the permitted use, you will need to obtain permission directly from the copyright holder. To view a copy of this licence, visit http://creativecommons.org/licenses/by/4.0/.

(c) The Author(s) 2022 